\title{
Research and development of virtualization in Wireless sensor networks
}

\author{
M. Sandeep Kumar", Prabhu. J* \\ \# School of Information Technology and Engineering, Vellore Institute of Technology, VIT University, India \\ Associate Professor, School of Information Technology and Engineering, Vellore Institute of Technology, VIT University, India \\ E-mail: sandeepkumarm322@gmail.com
}

\begin{abstract}
Virtualization is foundational for applying both cloud computing and big data. It provides the basis for many platform attributes required to access, store, analyze, and manage the distributed computing components in big data environments. Virtualization also able to use in a wireless sensor network in Agriculture field. WSNs emerging approach in commercial applications like Healthcare, agriculture, Industries. Virtualization in sensor network offers more flexibility, cost, effective solution; promote diversity, security, and manageability. In this chapter describes WSNs in agriculture domain, issues, challenges, etc. Also describes virtualization technology, techniques, and tools.
\end{abstract}

Keywords - WSNs, Agriculture, Big Data, Virtualization, Sensor.

\section{INTRODUCTION}

Currently most exciting topic worldwide is wireless sensor networks (WSNs). Most of application and business opportunities perform daily life with WSN business increase $\$ 0.45$ billion in 2012 to $\$ 2$ billion in upcoming years at 2022.WSNs mainly described based on a network with small devices as sensor nodes; it spatially assigned and perform cooperatively in connecting information group by monitoring field by wireless links. Data are collected based on various nodes sent to the sink, it may neither used as data placed or placed on another network. WSNs technology provides more benefits over previous network solution like less cost, scalability, reliability, accuracy, flexibility and easy to spot with a more comfortable range of application. Agriculture practices based on sensors and network only. Precision farming, Smart agriculture, intensive agriculture, variable rate technology based on sensor-based farming and process are similar to each other. Many researchers' analysis and come to know that issues arise from sensors and networks. Some of the problems like energy constraints, usage of small memory, data security, and restriction in power are issues form a sensor network, and most of the research identifies a solution.

\section{BACKGROUND}

Md.Motaharul Islam et al. (2012) describes the novel approach for utilizing the significant data using WSN resources in the sensor in virtualization platform. It represents sensor virtualization architecture, challenges, opportunities that focus on research field in sensor network virtualization.

Sunil N.more et al. (2002) describes an overview of various WSN technologies that used in the agriculture field. It offers information regarding WSN, and its standard technology namely types of WSN framework, wireless communication, different wireless sensors with a particular application and a case study of WSN.

Extraction of the sensor and its efficient usage to solve agriculture in the field. A difficult task for displaying user's unavailability in increase information. Aqeel -ur- Rehman et al. (2014) describe the requirement of wireless sensors in agriculture, WSN technology, its application in various need of farming by review document in the existing framework in agriculture application.

Manijeh Keshgary et al. (2012) describe two topologies of WSN for producing automated monitoring for precision agriculture. The first topology represents each sensor node determined in a random way for evaluating topology based on OPNET Modeler. In this model, sensor nodes data's gather depends on water level, gate arrangement, rainfall and soil moisture. Further real-time information's communicated to the sink node via Wi-Fi. Delay, throughput, and load are parameters used to set the performances.

Wen $\mathrm{Hu}$ et al. (2010) represent the application of WSN technology for a long time and large-scale environmental monitoring.In this work, we describe a real-time example of agriculture, investigate issues, challenges that meet the requirements for farmers in information collection system. 
Reliability and productivity are two main essential aspects that designed for selecting both system hardware and software.

Mohd Fauzi Othman et al. (2012) examine an overview of wireless sensor network application based on the environmental monitoring.It associates to implement perfect monitoring system, various needs to be followed. From this case study of the monitoring system, we have another way to represent the conventional approach that has been used to monitor the environment. This approach will offer more improvised system performance for convenient methods that satisfy entire functional requirements.

Xiaoqing Yu et al. (2013) describe hybrid wireless sensor network framework for agriculture. In this system, we compared between a hybrid WSN and a terrestrial wireless sensor network; it conveys information in the soil. Experiment analysis using soil that consists of $55 \%$ and $35 \%$, silt, and $15 \%$ clay, it had specific density of $2.6[\mathrm{~cm} \rrbracket \wedge(-3)$ and $1.5 \mathrm{~g} / \square \mathrm{cm} \triangle \wedge 3$ respectively. This experiment evaluated based on several soil moistures and three signal frequency measures. Finally result shows that the radio signal path loss is less for low frequency and moisture in soils.

Tamoghna Ojha et al. (2015) describe a clear overview of deployment of WSN in different farming application present in Indian soil. We also investigate benefits, issues, and solution in WSN, while finding the factor for enhancing and further research direction in WSN with new age technology.

Raul Morais et al. describes data acquisition platform for a gathering of solar, wind, water information by using ZigBee network. Zhang et al. (2007) demonstrate greenhouse wireless monitoring system; it could be more realistic in directing WSN based on ZigBee technology. Li and Liu et al. represent greenhouse monitoring framework that applied by WSN for controlling terminal of the system.An overall structure designed by ARM0 and Linux based on usage of the data receiver, real-time overview and preserving data in a greenhouse environment. The Main control terminal used for transmitting with remote management center by GPRS. The entire data acquisition from greenhouse platform based on WSN only.

Zhang.Z et al. (2017) demonstrate agriculture information collection framework that used for monitoring agriculture platform based on ZigBee technology. This context more relies on collecting soil information with high feasibility. This structure not only useful in resolving issues of low agricultural irrigation, water usage rate based on the presence of soil moisture gathered by WSN nodes. Water -saving, enhancing effective irrigation management based on soil moisture sensors. Further ZigBee technology more practices in industry for collecting information, detecting of blood pressure, temperature and heart rate information in the medical field.

Haiyuan sun et al. (2017) describes the practical examination of mobile software development technologies and Zigbee communication network. This system performs more compact and flexible ios based client, for considering benefits of Model view model. Message digest algorithm and Rivest Shamir Adleman algorithm that is integrated to secure information security by using encryption technologies. By comparing with specific marking framework, this system offers more accuracy in gathering information from WSN.
Rubella et al. (2017) describe crop monitoring framework based on the wireless sensor network. Currently, IOT plays a crucial role in promoting agriculture information. ARM processors combined with sensors like temperature, humidity, water level utilized for agriculture monitoring and crop production. The crops details monitored and upload data are in the cloud on IOT platform. Input material for saving, efficiency, productivity, profitability for improving production system for a farmer in controlling the production of agriculture.

Zulkifli C.Z et al. (2017) describes RFID (Radio Frequency Identification) technology and WMSN (Wireless Mess Sensor Network) for agriculture field, crop monitoring, plant.Wiring, piping cost, installation, management for a large area for reducing the WMSN. The use of technology in agriculture required for improving production for reducing labor cost and water needs. Hence WMSN technology determines well in most of the technology in developing the current irrigation frameworks. Continuously improvement and correct rate that afford for a large dataset for WMSN application by using soil moisture sensors.

Balakumar et al. (2017) demonstrate various possibilities in combining with distributed clustering mechanism with real-time applications. Moreover this technique that combined with lifetime sensor node that has utilized in remote and critical applications.

Mondal.A et al. (2017) represents detailed design level of implementing the low-cost WSN for agriculture domain.The successful construction for performing the detail designed node for water present in the soil for low cost in WSN. The Selection of the component, combined with building the prototype, firmware, hardware for implementing this work. The transmission of data in APL mode improved based on its reliability of the network. But the packets well reduce less around $0.1 \%$ by implementing static routing algorithm based on the particular scenario.

Arun.M Patokar et al. (2018) construct precision agriculture framework for monitoring the metrics that need for the crop. For producing precision agriculture, WSN platform affirms in making acquiring, processing information, recording the data node. This system provides low cost based on the type of system required to enhance the efficiency of resource used for improving performance in production. The Production of crop directly access and controlled by smart phone's; it affords more flexibility, user-friendly, fast in processing.

Construction Of Virtualization Of Wireless Sensor Network

Design aim in VSN (Virtualization sensor network)

The primary objective of performing the virtualization in a sensor network offered by many researchers. Some of the issues behind sensor network virtualization, each stage need to fulfill their criteria

Flexibility

Flexibility constructed based the adjusting change occur from external, and VSNs offers some problems in elasticity. VSN provides more freedom in each feature of sensor networking. Sensor virtualization network service provider need to generate shapes of virtual sensor network topology. It gives more flexible routing and functionality in altering control protocols that independently placed in the physical 
sensor network with previous SVNSPs (Sensor Virtualization Network Service Provider).

Network Heterogeneity

It is most necessity issues in constructing the VSNs. It mainly based on two aspects like i) Heterogeneity-based on sensor Network technology with various sensor nodes. ii) Each point to point SVNSP designed at the top of the heterogeneous mixture based on SInp, it also called heterogeneous. SVNSPs need to perform across various domains, point to point VSNs without the requirement of any particular solution. It mainly based on infrastructure it can assist different protocol, an algorithm for performing with various SVNSPs. More Heterogeneity of end user devices needs to consider.

Isolation

Sensor network Virtualization needs to assure isolation with previous VSNs to enhance in fault -tolerance, security, and privacy. Sensor network protocol is unerect to misconfigure; an error occurs in implementation. Sensor virtualization need assure the misconfiguration on single VSNs even though it exists with same physical part of the sensor network. Device abstraction is needed to move further with effective software isolation; the various instance needs to be unmodified with sensing application by using the virtual sensors in applying with the physical sensor. Mostly strong isolation is enhanced their performance when each user's performance with shared function device resources. Sharing Guarantees provides fewer fractions in sensor resources.

Manageability

Central important part of constructing VSN is to maintain the VSN applications. VSNs offer each SVNSP stay independent in a physical sensor network. By splitting SVNSPs from SInPs, sensor network virtualization needs to standard network in maintaining each step. It describes each layer in network architecture needs offer entire, point to point control over NSNs to SVNSPs. Moreover, it requires coordination in the various regions in previous WSN domains.

\section{Scalability}

Sensor networks are the more specific principle of sensor network virtualization. We get Scalability from indispensable component equations. SInPs (Sensor Infrastructure provider) are scalable assist in more number of coexisting VSNs without any changes in the performance. In the construction of large-scale sensor network, it requires to perform the VSNs scalability; any changes can be made or efficiently in implementing the physical sensor network.

Stability and Convergence

Isolation assures the faults of single VSN, it will not affect with other VSNs, in causing some errors, misconfiguration based on physical network, it also unstable with the sensor network and virtualization platform. Due to instability occurrence in SInPs, it lies instability for the entire host in VSNs. Mostly VSNs assure the balance of sensor virtualization platform in some scenario. Uncertainty may affect with VSNs required to perform successfully in converge of stability.

Programmability

Programmability provides more assurance in both flexibility and manageability. Indispensable require in virtual sensor network component as programmability. By using programmability; SVNSPs perform protocol place in services.
Two need questions to satisfy: Whether programmability requires allowing? Then how to achieve? Solutions are merely comfortable, practical and more secure of some duration. For distributing resource constraints in a sensor node, programmability offers more research gaps in VSNs.

Legacy support

It mainly concerns with placing the new approach or technology. We can easily integrate legacy with sensor network virtualization in assisting with previous WSN domain like VSN, in gathering sensor networks due the efficiency are still research challenges. Most of the researchers concentrate on design only.

\section{Some Of The Challenges While Constructing Vsns \\ Interfacing}

SVNSP make use of physical resources from single to multiple environments, it offers in performing virtual sensor network. SInP necessity needs to provide the interface that allows service providers for connecting and giving the needs. Interoperability is similar to an interface that needs to recognize the standards requirements in performing sensor virtualization as a request to various nodes, links of virtual sensors with some attributes. Monitoring interface is a perfect example of sensor web design.

Managing limited resource

Resources allocation offers sensor network virtualization platform, consists of both static and dynamic allocation of the node. Links of virtual sensor connects with physical nodes that as paths called virtual sensor network embedding. In this constraint of nodes, links offer in decreasing the NP-based issues. When an entire network request to determined in advance. Storage, power consuming, less battery power are some issues in maintaining. More efficient research need an application from the virtual network to real WSN requires. Moreover, it will be accessible and useful in constructing the virtualization in sensor nodes.

Resource Discovery

Resource Discovery allocates requests from various virtual sensor providers. The environment can perform topology sensor network in maintaining the vital sensor network components. It also shares the crucial information in determining the links between the network and even installation of the virtual sensor network.

Quality of service and experience

Quality of service mainly used for sorting the whole virtualizations. It evaluates the network operator's agreement like noise, decreases packets. Meanwhile, the experience assessing sensor network for fulfilling the various application stages based on user's needs. Need to ensure that significant challenges are in VSNs.

Security

Security offers data from the physical sensor node to various user application stages. In large scale, WSNs data are offers in physical sensors are the most necessary. Misuse the process effect in users level application, and VSNs need to offer both security and authentication control.

Resource Scheduling

The virtual sensor network is the providers that specifically assure the characteristics of the virtual node with the bandwidth to assigns its network. Virtual sensor routers are service providers that may request to ensure the limited 
processing packet in CPU for particular storage needs and also provides the memory size.

The virtual sensor connects for providing request range from most excellent service in combining with fix loss, and delay characteristics to recovered from physical links. It affords with assurance in producing the illusion in remote and placed network services for the single service provider.It required infrastructure need to deployed proper scheduling algorithm for an entire component of the sensor network. By Previous framework virtualization offers scheduling process like CPU, Storage, memory, network interface, it runs on each VM to host sensor node. Sensor network Virtualization may be enhanced control for performing the resource scheduling placed on the physical platform.

Admission Control and Resource Usage Policy

Sensor platform offers more secured that resource will not be part of Provisioned to transfer QoS support. It allows determining comprehensive accounting and performance in controlling the admission algorithm, in giving more assurance resource assigned to virtual sensor networks. It will not be more physical storage lies on physical sensor networks. Moreover, will not permit dynamic to rescale in allocating resources. To avoids the constraints iniquity by virtual sensor networks. Its controls by placing the service providers, will not overflow the enormous resource assign to them by using both direct and indirect process.

Naming and Addressing

By essential heterogeneity in naming and addressing outlines of the previous process. The Main challenges of sensor network virtualization like virtual sensor network, point to point connection, communication between universal. Moreover, various stages of application users at the same time communicate with various virtual networks by many platform providers by different techniques in processing separate service. It affords with incorporating heterogeneity in many shapes are some of the issues in sensor network virtualization. Some recent research projects are split with their identities, end host to physical and logical place to add some need stages in the new process.It also provides standard finding space to offer communication accurately without striking down both physical and virtual sensor network. Moreover, will not be a physical process because of unrestrained memory requires. The Main essential research gaps in naming, addressing in identifying the worldwide communication that able to connect with the federation sensor system.

Virtual sensor network operations and management

Some of the specific challenges in sensor network virtualization like Operation and management. By splitting accountability and duty among various users in sensor network virtualization platform. It provides more maintenance in decreasing process of error. By controlling both proactive and reactive process to enforce responsibility in hosting virtual sensor networks. Significance in flexibility needs to be described in various stages of sensor network operation centers to brilliant agents for sensor network components. Virtual sensor network operation can single service providers in configuration, maintain, accessing the virtual sensor networks with regardless of others. From virtual sensor network, it covers part lies in physical sensor networks, the application needs to improve with aggregate data from many places, frequently from conflict, maintain process analyze the users in sensor platform providers. Average layers of abstraction in continuing management software's provider with a more efficient solution. Most of the loss lies on physical sensor network elements that get from failures of cascading in virtual sensor networks; it straightly connects the host to the parts. For example, physical connection failures to provide results in whole virtual connection by passing it. Corresponding physical sensor node failure may need to repeat in installation for a complete service provider in custom software. Some of the failure like detection and isolation and also well with prevention and recuperation in the stable state are the some of the research gaps.

\section{Wsns USED IN AGRICULTURE DOMAIN}

Necessity of using WSNs

Ability in decision making

It provides many hops in nature. In the vast region, features need to improve in energy efficiency with the whole network and also improve in lifetime duration. Some of the features like multiple sensor nodes combined themselves and got the result.

Dynamic topology configuration

For preserving the power of the battery, sensor node acts them as a sleep mode in much time. By applying topology management approach, sensor nodes cooperate in decision making, in reducing network duration. The network topology performed with less number of nodes as it stays in active mode.

Fault tolerance

The Main challenges in performing the WSNs in sensor node called fault -Prone. Below is some scenario, unplanned nodes placed for getting an advantage in network partitioning and also change to the entire performance of network are disturbed. Moreover, measure from sensor nodes may be selforganized in the network topology.

Context-awareness

It mainly consists of sensor data based on physical and environmental components. Sensor nodes benefit knowledge on covering context. A decision on sensor nodes based on context-aware.

Scalability

WSN protocol constructed for performing the network regardless of its size and number of count. The Features of WSNs are essential for most of the applications.

Node heterogeneity

WSNs are frequently assumed to determine homogeneous sensor placed in devices. Computation power, memory, sensing ability, transceiver unit, and movement capability are some real scenario based on methods in different processing.

Tolerance versus communication failure in criticizes platform conditions

Vast areas in agriculture domain, WSNs uncomforted in criticize platform conditions. WSN protocol stacks some of the techniques that have phenomenon failure in communication network caused because of surrounding effects.

Autonomous operating nodes

Some potential features of WSNs are self-reliant operating mode and adaptiveness. In an application like agriculture has some specific features plays a crucial role in performing well in operation modes.

Information security 
WSNs convey raw data in concerning parameter of agriculture field. It assures the security of sensing data. WSNs offers process control mechanism; anomaly detection limited to unauthenticated customers.

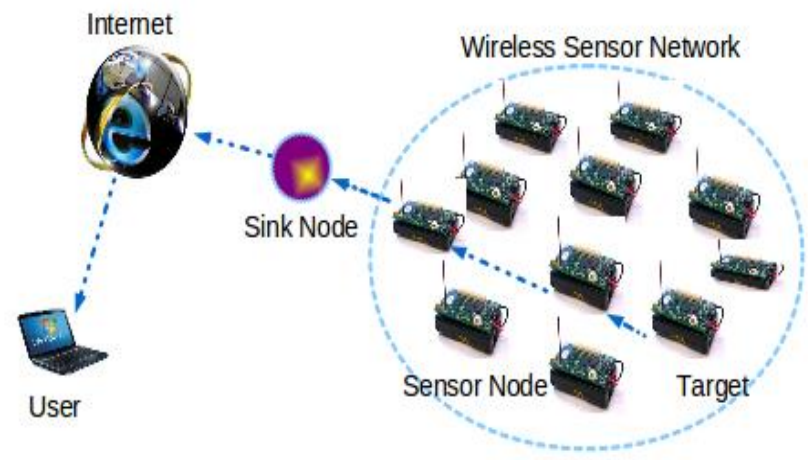

Fig. 1 Process of WSNs

Requirement in using Sensors in Agriculture

The sensor collects data from physical and environmental attributes.Actuators perform based on feedback and situations. Sensor accumulate used of finding the people, locations, objects are assigned as context. Context acquisition offers to need contribution over time variants attributes.

It requires various needs in agriculture domain like grouping data like weather, crop, and soil.The Maintains of various lands and diverse crop in one field. Need crops for numerous regions in different weather and soil condition. We use proactive solution instead of reactive solutions. Moreover, it requires both parallels, distributed processing. Wireless Sensors and actuator need to gather the data in various situations based decision making.

Types of sensor used in Agriculture Domain

In this section, we explicitly describe various types of sensor used in agriculture field like soil, weather, plant, and environment. In table 1, 2, 3, 4; represent the different sensor utilized in this area.

TABLE 1

SENSOR USED IN AGRICULTURE (SOIL)

\begin{tabular}{|l|l|l|l|l|l|}
\hline Sensors & $\begin{array}{l}\text { Tempe } \\
\text { urature }\end{array}$ & Moisture & $\begin{array}{l}\text { Flow } \\
\text { of } \\
\text { water }\end{array}$ & $\begin{array}{l}\text { Conduc } \\
\text { tivity }\end{array}$ & Salinity \\
\hline EC sensor & + & + & + & + & + \\
\hline Hydra Probe soil & + & + & + & + & + \\
\hline MP 406 soil & + & + & - & - & - \\
\hline 107-L temperature & + & - & - & - & - \\
\hline $\begin{array}{l}\text { ECRN-100 high }- \\
\text { REC rain }\end{array}$ & - & - & + & - & - \\
\hline
\end{tabular}

TABLE 2

SENSOR USED IN Agriculture (WEATHER)

\begin{tabular}{|l|l|l|l|l|l|}
\hline Sensor & $\begin{array}{l}\text { Tempe } \\
\text { rature }\end{array}$ & $\begin{array}{l}\text { Wind } \\
\text { Speed }\end{array}$ & $\begin{array}{l}\text { Wind } \\
\text { Direction }\end{array}$ & $\begin{array}{l}\text { Humi } \\
\text { dity }\end{array}$ & $\begin{array}{l}\text { Atmos } \\
\text { pheric } \\
\text { pressure }\end{array}$ \\
\hline $\begin{array}{l}\text { Weather } \\
\text { Sensor }\end{array}$ & + & + & + & + & + \\
\hline $\begin{array}{l}\text { SHT71 } \\
\text { (Humidity and } \\
\text { temperature) }\end{array}$ & + & + & + & - & - \\
\hline
\end{tabular}

\begin{tabular}{|l|l|l|l|l|l|}
\hline $\begin{array}{l}\text { 107-L } \\
\text { temperature }\end{array}$ & + & - & - & - & - \\
\hline $\begin{array}{l}\mathrm{CM}-100 \\
\text { compact }\end{array}$ & + & + & + & + & + \\
\hline $\begin{array}{l}\text { Cl-340 hand- } \\
\text { held } \\
\text { photosynthesis }\end{array}$ & + & + & - & - & - \\
\hline
\end{tabular}

+: Available -: Not available

TABLE 3

Sensor Used IN Agriculture (Plant)

\begin{tabular}{|l|l|l|l|l|l|l|}
\hline Sensor & Moisture & $\begin{array}{l}\text { Temper } \\
\text { ature }\end{array}$ & CO2 & $\begin{array}{l}\text { Photo } \\
\text { synthesis }\end{array}$ & $\begin{array}{l}\text { Hydro } \\
\text { gen }\end{array}$ & wetness \\
\hline $\begin{array}{l}\text { Leaf wetness } \\
\text { sensor }\end{array}$ & + & - & - & - & - & - \\
\hline $\begin{array}{l}\text { 237-L, leaf } \\
\text { wetness } \\
\text { sensor }\end{array}$ & + & + & - & - & - & + \\
\hline $\begin{array}{l}\text { LW 100,leaf } \\
\text { wetness } \\
\text { sensor }\end{array}$ & + & + & - & - & - & + \\
\hline $\begin{array}{l}\text { SenseH2 } \\
\text { hydrogen } \\
\text { sensor }\end{array}$ & + & + & - & - & - & + \\
\hline $\begin{array}{l}\text { TPS-2 } \\
\text { portable } \\
\text { photosynthesis }\end{array}$ & + & + & + & + & - & + \\
\hline $\begin{array}{l}\text { CI-340 hand - } \\
\text { held } \\
\text { photosynthesis }\end{array}$ & + & + & + & + & + & + \\
\hline $\begin{array}{l}\text { PTM-48A } \\
\text { photosynthesis } \\
\text { monitor }\end{array}$ & + & + & + & + & & + \\
\hline
\end{tabular}

TABLE 4

SENSOR USED IN AGRICULTURE (ENVIRONMENT)

\begin{tabular}{|c|c|c|c|c|c|c|c|}
\hline Sensor & $\begin{array}{l}\text { Humi } \\
\text { dity }\end{array}$ & $\begin{array}{l}\text { Te } \\
\text { mp } \\
\text { era } \\
\text { tur } \\
\text { e }\end{array}$ & $\begin{array}{l}\text { Atmo } \\
\text { spher } \\
\text { ic } \\
\text { press } \\
\text { ure } \\
\end{array}$ & $\begin{array}{l}\text { Wind } \\
\text { speed }\end{array}$ & $\begin{array}{l}\text { Direc } \\
\text { tion }\end{array}$ & $\begin{array}{l}\text { Rainf } \\
\text { all }\end{array}$ & $\begin{array}{l}\text { Solar } \\
\text { radia } \\
\text { tion }\end{array}$ \\
\hline $\begin{array}{l}\text { WXT 520 } \\
\text { compact } \\
\text { weather } \\
\text { station }\end{array}$ & + & + & + & + & + & + & - \\
\hline $\begin{array}{l}\text { CM-100 } \\
\text { compact } \\
\text { weather } \\
\text { station }\end{array}$ & + & + & + & + & + & - & - \\
\hline $\begin{array}{l}\text { Met } \\
\text { station } \\
\text { one } \\
\text { (MSO) } \\
\text { weather } \\
\text { station }\end{array}$ & + & + & + & + & + & - & - \\
\hline $\begin{array}{l}\text { All -in- } \\
\text { one } \\
\text { (AIO )we } \\
\text { ather } \\
\text { sensor }\end{array}$ & + & + & + & + & + & - & - \\
\hline $\begin{array}{l}\text { XFAM- } \\
115 \\
\text { KPASR } \\
\end{array}$ & - & - & - & & & - & - \\
\hline $\begin{array}{l}\text { RM } \\
\text { Young } \\
\text { (Model } \\
5103 \text { ) }\end{array}$ & - & - & - & + & + & - & - \\
\hline $\begin{array}{l}\text { Met one } \\
\text { series } 380 \\
\text { rain gauge }\end{array}$ & - & - & - & - & - & + & - \\
\hline $\begin{array}{l}\text { RG13/RG } \\
13 \mathrm{H}\end{array}$ & - & - & - & - & - & + & - \\
\hline
\end{tabular}






TABLE 5

PERFORMS OF SENSORS

\begin{tabular}{|l|l|l|l|l|}
\hline Item & Condition & $\begin{array}{l}\text { Amount of } \\
\text { water level }\end{array}$ & Min & Max \\
\hline & Sensor in dry soil & High & $0 \%$ & $30 \%$ \\
\hline Output & Sensor in humid soil & Medium & $30 \%$ & $70 \%$ \\
\hline & Sensor in water & Low & $70 \%$ & $85 \%$ \\
\hline & Sensor in water & No & $85 \%$ & $95 \%$ \\
\hline
\end{tabular}

TABLE 6

COMPARISON OF WSN TECHNOLOGIES

\begin{tabular}{|l|l|l|l|l|}
\hline & Zigbee & Bluetooth & Wibree & WiFi \\
\hline $\begin{array}{l}\text { Frequency } \\
\text { band }\end{array}$ & $2.4 \mathrm{GHz}$ & $2.4 \mathrm{GHz}$ & $\begin{array}{l}2.4 \\
\mathrm{GHz}\end{array}$ & $2.4 \mathrm{GHz}$ \\
\hline Range & $30 \mathrm{~m}-1.6 \mathrm{~km}$ & $3-300 \mathrm{ft}$ & $\begin{array}{l}\mathrm{Up} \mathrm{to} \\
10 \mathrm{ft}\end{array}$ & $100-150 \mathrm{ft}$ \\
\hline Data rate & $250 \mathrm{hbps}$ & $1 \mathrm{Mbps}$ & $1 \mathrm{Mbps}$ & $11-54 \mathrm{Mbps}$ \\
\hline $\begin{array}{l}\text { Power } \\
\text { consumption }\end{array}$ & Low & Medium & Low & High \\
\hline Cost & Low & Low & Low & High \\
\hline Protocol & DSSS,CSMA/CA & FHSS & FHSS & $\begin{array}{l}\text { DSSS } \\
\text { /CCK,OFDM }\end{array}$ \\
\hline Security & 128 bit & $\begin{array}{l}64 \text { or } 128 \\
\text { bit }\end{array}$ & 128 bit & 128 bit \\
\hline
\end{tabular}

ZigBee, Bluetooth, Wibree, and WiFi are some of the wireless communication techniques that have part of various sensor network based on research aspects. This technology has the different ability and its properties that are having complemented. Clear and brief comparison in given table 6 . Some Of The Issues In Wireless Sensor Networks In Agriculture Field

Wireless sensor networks mainly based on flexible and autonomous, it has opportunities to design with previous application domain like remote sensing and optimized solution. Wireless sensor network technology has many problems that required dealing with long-term availability framework. Sensor nodes dictate design and development the energy consumption, communication, protocols, deployment. In many scenario farming placed with various resources, nature of land and different climate.

Energy Consumption

Sensor nodes deployed in a wireless sensor network, consists of event detection, data processing, and transmission are the responsibility of WSNs. In some case, the multi-hop network has a task in node and data routing. Each work requires energy consumption. Node is based on the restrict the perfect source for Lithium cells. Sensor node lifetime mainly based on battery life duration. By placing required energy management approach in hardware and software, battery life needs to enhance more length. Use of renewable energy sources like solar power and kinetic energy required to adjust, when less expensive result. However in some case of agriculture domain sensor simultaneously recharge when battery altered their places and process usually well.

Data acquisition, sampling, and transmission
Energy is spent in every data samples, process and transmits. Optimized data are gathered with sample rate when it required being programmed and not only need data, but energy also not spent unnecessarily. Frequently data acquisition makes a large number of packets that are transmitted and discharge the battery quickly. In agriculture domain, the sampling rate is not higher, and it will make a change depends on types of crop used in resources.

Fault tolerance

Sensor nodes are deployed in criticize platform, unerect to a physical cause, block, and interference. To preserve the reliability of a WSN, failure of sensor node need not cause the entire process of the network. Some technique placed on improving the fault tolerance in a system like the use of sensor network, reprocessing sensor network and over processing sensor region.

Size and Housing in sensor nodes

It required being too small and needed to distribute. Some of the critical factors like heat, rain, physical misbehavior by human or animals.

\section{Sensor placement}

Sensor node placement is an essential problem in constructing the network topologies process using parameter comprising the sensor. It required to creating in designing and performing smartly when WSN need to established in reliability and autonomously. Sensors are need covered and placed in position in measuring parameter without the need for hindrance. Example sensor light is deployed in height and neglected the close plant leaves. Accurate measures put in ground-based on the level of water and moisture content on the ground. Deallocate of the sensor in various positions based on durable wind and water currents, need to place appropriately in required form with afford nodes.

Some Of The Essential Application In Agriculture And Farming Using WSNs

Irrigation management system

Now a day's agriculture needs better irrigation framework in optimizing the use of water in the farming process. Required structure in groundwater level as an alarming reduction. Cost effective and water usage are the some of the micro irrigation approaches.Moreover, micro irrigation is better based on soil formation and environment. WSNs placed with coordinating techniques.

Farming Monitoring framework

Now different useful structure and device make use in farming. A better context is to maintain this method to secure the whole operation, process automated for farming. Remote monitoring framework assists in better management in colossal agriculture domain. The input of traditional data like satellite images, weather forecast, and performance system will be better.

Pest and control disease

Usage of pesticides and fertilizer assist in improving the crop quality and cost Control. Moreover, utilization of pesticides can control the occurrence of the crop in pests. For analyzing the environment like temperature, humidity, and speed of the wind. WSNs can be monitor and analysis the events over a field of interest.

Horticulture

Horticulture mainly based on low scale or less intensity farming. Cultivation productions, distribution, usage of 
flower, fruit, and ornamentals are handling in Horticulture.The primary research describes in this section about greenhouse and Horticulture. The Present state process of plant nursery helps based on usage of sensor network analyzed in temperature, humidity, soil moisture, and temperature. It also used for the handle and identified the plant diseases.

TelosB sensor motes and Ech2o soil moisture sensor are introduced for context-aware irrigation activity framework. $30 \%-50 \%$ of conservation of water analyze in green area irrigation by using field test.

Growth is to decrease the cost of irrigation activity using sensor node and WSAN protocol. Light, humidity, soil moisture sensor are irrigation monitor using five sensor nodes. Nodes are constructed based on Atmel ATMega 64L, AVR microcontroller referable afford with six sleep modes like Idle, ADC, Noise Reduction, Power-save and down, enhance, Stand-by. Some of less power feature consumption like XBee and OEM RF module. Some advantage provides for power consumption; cost properties are construct instead of the size of sensor mode.

\section{Viticulture}

The essential usage of sensor network performs in Vineyards. They briefly explain the variety of sensor network configurations and its application based on various priorities in the vineyard. It takes more than six months to construct with 65 nodes and multi-hop WSN. Gathering of data mainly used of two need aspects in the production of wine like heat summation and essential frost damage.

It provides feasibility in ZigBee, we using remote sensor network called as MPWiNodeZ, propose for precision viticulture. Solar power, wind power, Hypro power are batteries used for recharging the harvest energy platform.

\section{Green House}

Monitoring environment and greenhouse controlling are essential aspects but not depends on the natural agent. By collecting both in and out climate data in the greenhouse for much solar power, wireless acquisition station (SPWAS) used to construct wireless data acquisition network. Connection using RF links used as a base station, up to 32 SPWASs. The Collection of climate data successfully without any errors. Growth in both hardware and software acquisition framework depends on Bluetooth technology. Temperature and relative humidity take a measurement.

Future Aspect of WSNs

The essential application used in WSNs in agriculture and farming. The present scenario works in irrigation management, Greenhouse, Viticulture, Horticulture, pest control disease, farming monitoring framework.

Some of the factors needs to improve in WSNs in future Cost

Provides of less cost result ever suitable for enhancing the scope and stretch of the application.

Autonomous operation

The Longtime result of the provision for independent operation needs for future purpose.

Intelligence

The changes in the result in providing various challenges for preserving the energy in real time process.

Portability
Portability essential using in the application and framework like Sip (System in package) and SoC (System on Chip) are technologies applied for consideration.

Low maintenance

Low maintenance potentially used for construct framework it needs to the less maintained effort. It performs a long time based on some average cost.

Energy-efficiency

To get assurance in enhance lifetime with the autonomous operation, result required to get improved energy efficient incorporating with intelligent algorithms.

Robust architecture

The current arising application needs to assure the required operation for robust and fault tolerant structure.

Ease of operation

The application requires using easy and simple to manage. Mostly non - the technical person will be the end-user for the applications.

Interoperability

It varies between the different component and communication technologies for improving the entire functionality of the framework.

Some additional challenges in the global scenario and Indian scenario with WSNs in agriculture. Listing some potential problems in Indian agriculture.

Cost: Sensor cost is higher, as it combined with an application like LMISs.

Variable climate and soil: Most significant part in constructing the WSNs in agriculture in India varies in temperature, types of land. Some parameter needs to perform in function properly with other location also.

Segmented land structure:

Some specific challenge in Indian agriculture using WSNs like irrigation management.

Average farmer requirement

Average land having per farmer is less than global status because of the necessity of smaller and personalized framework.

Overall plan

Structure of segment land and farmer need to get success in automatic farming and agriculture.

Future Application

In a Current scenario more advance new technique and concepts like sensor cloud technology, big data analytics, Internet of thing (IoT).

Sensor cloud computing

Sensor cloud computing mainly based on Land, WSN application combined with cloud computing. It connected system in getting an advantages WSNs for better power and storage. Moreover, sensor cloud provides better data management and control accessing in more resource usage. Some essential application used in agriculture domain like

- $\quad$ Cloud providers in storing the spatial difference in soil and environment platform with various season required to arises.

- Mobile sensor cloud services used for managing the crop monitoring and yield prediction.

- $\quad$ Construction of sensor cloud manages vast field using smart irrigation framework. 
- To construct sensor cloud control environment framework for breaking season production of Big data analytics greenhouse farming like vegetable and flowers.

Big data analytics applied to large data with many data types. Hidden correlations, unknown pattern, business trends.Disaster is possible techniques used in big data analytics.

- Construct growth of crop and managing disease model depends on farm data.

- Web analytics service used by the farmer for better information about agriculture.

- For large agriculture field, the farmer used equipment control framework.

- Decision affords service for better productivity in the crop at low cost with climate information and largescale agriculture.

- Government and industries perform optimal policy in determining big data analytics.

Internet of things

IoT provides an essential result with a different application. Some critical IoT based agriculture application like

- $\quad$ RFID tags used for cost-effective and agriculture supply chain management.

- Animal process monitor by remote.

- User rate and time depend on control of remote and duration for spraying pesticide.

- $\quad$ Leak detection and remote water flow are access and maintain the vast field for the supply of water.

\section{CONCLUSION}

We describe Virtualization using wireless sensor Network in Agriculture field. WSNs emerging approach in commercial applications like Healthcare, agriculture, Industries. Virtualization in sensor network offers more flexibility, cost, effective solution; promote diversity, security, and manageability. We describe the construction of virtualization in WSNs with some necessity like Network heterogeneity Isolation, Management, Scalability, Stability and convergence, programmability, legacy support. Some challenges in implementing VSNs such as Interfacing, managing limited resources, resource discovery, quality of services and experience, security, resource scheduling, Naming and addressing, Virtual sensor network operation and management, etc. Analysis performance of WSNs in agriculture based on Dynamic topology configuration, fault tolerance, context awareness, scalability, Node heterogeneity, autonomous operating nodes, and information security and explains with the sensor used in agriculture in soil and weather. The parameter used in the soil like temperature, moisture, the flow of water, conductivity, salinity and utilized in weather like temperature, wind speed, direction, humidity and atmospheric pressure. WSNs has some issues in performing in agriculture domain like energy consumption, Data acquisition, sampling and transmission, fault tolerance, size and housing in sensor nodes, sensor placement and specific application in agriculture farming by WSNs such as Irrigation management, Farming monitoring, Pest control, horticulture, viticulture, greenhouse. Future aspect of WSNs in Big data analytics, IOT, Sensor cloud computing.

\section{REFERENCES}

[1] Islam, M. M., \& Huh, E. N. (2012). Virtualization in Wireless Sensor Network: Challenges and Opportunities. JNW, 7(3), 412-418.

[2] Keshtgary, M., \& Deljoo, A. (2012). An efficient wireless sensor network for precision agriculture. Canadian Journal of Multimedia and Wireless Networks, 3(1), 1-5.

[3] Othman, M. F., \& Shazali, K. (2012). Wireless sensor network applications: A study in environment monitoring system. Procedia Engineering, 41, 1204-1210.

[4] Ojha, T., Misra, S., \& Raghuwanshi, N. S. (2015). Wireless sensor networks for agriculture: The state-of-the-art in practice and future challenges. Computers and Electronics in Agriculture, 118, 66-84.

[5] Zulkifli, C. Z., \& Noor, N. N. (2017). Wireless Sensor Network and Internet of Things (IoT) Solution in Agriculture. Pertanika Journal of Science \& Technology, 25(1).

[6] Akyildiz, I. F., Su, W., Sankarasubramaniam, Y., \& Cayirci, E. (2002). Wireless sensor networks: a survey. Computer networks, 38(4), 393422.

[7] Hill, J., Horton, M., Kling, R., \& Krishnamurthy, L. (2004). The platforms are enabling wireless sensor networks. Communications of the ACM, 47(6), 41-46.

[8] Valente, A., Morais, R., Serodio, C., Mestre, P., Pinto, S., \& Cabral, M. (2007, October). A ZigBee sensor element for distributed monitoring of soil parameters in environmental monitoring. In Sensors, 2007 IEEE (pp. 135-138). IEEE.

[9] Youbing, Z. R. G. G. F., \& Chengfei, L. (2008). Realization of Communication in Wireless Monitoring System in Greenhouse Based on IEEE802. 15.4 [J]. Transactions of the Chinese Society for Agricultural Machinery, 8, 029.

[10] Kim, Y., Evans, R. G., \& Iversen, W. M. (2008). Remote sensing and control of an irrigation system using a distributed wireless sensor network. IEEE transactions on instrumentation and measurement, 57(7), 1379-1387

[11] Li, L., Li, H. X., \& Liu, H. (2009). A greenhouse environment monitoring system based on the wireless sensor network. Transactions of the Chinese Society for Agricultural Machinery, 9(40), 228-231.

[12] Corke, P., Wark, T., Jurdak, R., Hu, W., Valencia, P., \& Moore, D. (2010). Environmental wireless sensor networks. Proceedings of the IEEE, 98(11), 1903-1917.

[13] Yu, X., Wu, P., Han, W., \& Zhang, Z. (2013). A survey of wireless sensor network infrastructure for agriculture. Computer Standards \& Interfaces, 35(1), 59-64.

[14] Abbasi, A. Z., Islam, N., \& Shaikh, Z. A. (2014). A review of wireless sensors and networks' applications in agriculture. Computer Standards \& Interfaces, 36(2), 263-270.

[15] Rawat, P., Singh, K. D., Chaouchi, H., \& Bonnin, J. M. (2014). Wireless sensor networks: a survey on recent developments and potential synergies. The Journal of Supercomputing, 68(1), 1-48.

[16] Ojha, T., Misra, S., \& Raghuwanshi, N. S. (2015). Wireless sensor networks for agriculture: The state-of-the-art in practice and future challenges. Computers and Electronics in Agriculture, 118, 66-84.

[17] Zhang, Z., Wu, P., Han, W., \& Yu, X. (2017). A remote monitoring system for agricultural information based on the wireless sensor network. Journal of the Chinese Institute of Engineers, 40(1), 75-81.

[18] Sun, H., Zhang, J., Sun, G., \& Li, Y. (2017). Agricultural Traceable and Marketing System Based on iOS-Platform and Wireless Sensor Network. Journal of Computer and Communications, 5(06), 45.

[19] Rubala, J. I., Anitha, D., \& Student, P. G. (2017). Agriculture Field Monitoring using Wireless Sensor Networks for Improving Crop Production. International Journal of Engineering Science, 5216.

[20] Prabhu, B., Balakumar, N., \& Antony, A. J. (2017). Wireless Sensor Network Based Smart Environment Applications.

[21] Mondal, A., Misra, I. S., \& Bose, S. (2017, April). Building a low-cost solution using wireless sensor network for agriculture application. In Innovations in Electronics, Signal Processing and Communication (IESC), 2017 International Conference on (pp. 61-65). IEEE

[22] Patokar, A. M., \& Gohokar, V. V. (2018). Precision Agriculture System Design Using Wireless Sensor Network. In Information and Communication Technology (pp. 169-177). Springer, Singapore. 